\title{
Quantum computers move a step closer
}

\section{Successes at entangling three-circuit systems brighten the prospects for solid-state quantum computing.}

BY EUGE

\section{Y EUGENIE SAMUEL REICH}

uantum computing has made another advance along the path from theorists' darling to working device.

The concept depends on entanglement, a strange phenomenon in which the quantum states of spatially separated systems, called 'qubits', become intrinsically linked. The entanglement of two or more qubits sets up a 'superposition' of states in which calculations can run in parallel - in principle allowing a quantum computer to race through problems that it would take a classical computer eons to solve.

Such a quantum machine would require hundreds or even thousands of entangled qubits. The maximum reached so far is 12 , but some of the systems that researchers are working with, including those depending on the spins of ions, may be hard to scale up. In this issue of Nature, two research groups ${ }^{1,2}$ report progress on an alternative approach: entangling qubits made from superconducting circuits, a technology that is amenable to manufacture on electronic chips. "Superconducting qubits are one of the better candidates for building a quantum computer," says Daniel Gottesman, a quantum researcher at the Perimeter Institute in Waterloo, Canada.

The teams have achieved three-qubit entanglement in such a system, which is significant because three is the minimum number needed for quantum error correction - an essential attribute if quantum computers are ever to become practical. A quantum computer is susceptible to flipping its bits and losing information. Measuring bits to check their values part way through a computation would destroy the superposition. But entangling each bit with two extra bits makes it possible to check two of those bits for errors while allowing the calculation to go forwards in the third.

To construct their qubits, a team led by Rob Schoelkopf of Yale University in New Haven, Connecticut, used superconducting aluminium wires cooled to within a degree of absolute zero. The circuits were linked so that voltage

$\rightarrow$ NATURE.COM

Relativity comes down to Earth. go.nature.com/GTStxu and current oscillations flowing through each one would influence the others, and the entanglement was generated with

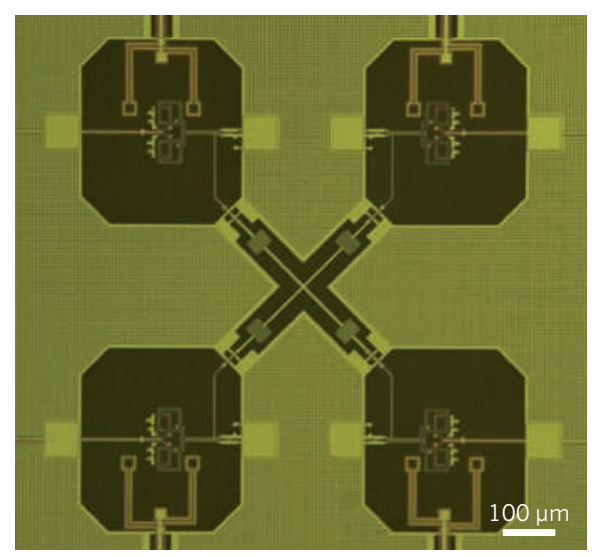

A circuit of four superconducting qubits. Scientists have succeeded in entangling three of these.

a sequence of microwave bursts that changed the states of the circuits. The result was a kind of entanglement called a Greenberger-HorneZeilinger (GHZ) state, in which the three qubits are in a superposition of all being zero, and all being one.

A second group, led by John Martinis of the University of California, Santa Barbara, also succeeded in creating the GHZ state, as well as a 'W state', in which the superposed states feature one qubit with a value of one and the other two with a zero.

Neither group has used their three entangled bits to run quantum error correction yet. But Schoelkopf emphasizes that his group has already run another type of algorithm using two-qubit entanglement ${ }^{3}$. He adds that a future challenge will be finding a way to lengthen the lifetime of the qubits, which lose their information within about 100 operations.

Emanuel Knill, an expert in quantum information science at the National Institute of Standards and Technology in Boulder, Colorado, isn't sold on the approach, noting that it will be difficult to control multiple qubits from outside a refrigerator. But he says he's happy to see that both groups prepared their quantum states with decent fidelities, meaning that the states are a good match to those the researchers intended to create. "The challenge," he says, "is to scale up the number of gates and qubits." -

1. DiCarlo, L. et al. Nature 467, 574-578 (2010).

2. Neeley, M. et al. Nature $467,570-573(2010)$.

3. DiCarlo, L. et al. Nature 460, 240-244 (2009). 